\title{
Existence of Solutions for a Modified Nonlinear Schrödinger System
}

\author{
Yujuan Jiaoo ${ }^{1}$ and Yanli Wang ${ }^{2}$ \\ ${ }^{1}$ College of Mathematics and Computer Science, Northwest University for Nationalities, Lanzhou 730124, China \\ ${ }^{2}$ School of Mathematical Sciences, Beijing Normal University, Beijing 100875, China \\ Correspondence should be addressed to Yujuan Jiao; jsjyj@xbmu.edu.cn
}

Received 8 April 2013; Accepted 18 June 2013

Academic Editor: Renat Zhdanov

Copyright (c) 2013 Y. Jiao and Y. Wang. This is an open access article distributed under the Creative Commons Attribution License, which permits unrestricted use, distribution, and reproduction in any medium, provided the original work is properly cited.

We are concerned with the following modified nonlinear Schrödinger system: $-\Delta u+u-(1 / 2) u \Delta\left(u^{2}\right)=(2 \alpha /(\alpha+\beta))|u|^{\alpha-2}|v|^{\beta} u, x \in$ $\Omega,-\Delta v+v-(1 / 2) v \Delta\left(v^{2}\right)=(2 \beta /(\alpha+\beta))|u|^{\alpha}|v|^{\beta-2} v, x \in \Omega, u=0, v=0, x \in \partial \Omega$, where $\alpha>2, \beta>2, \alpha+\beta<2 \cdot 2^{*}, 2^{*}=2 N /(N-2)$ is the critical Sobolev exponent, and $\Omega \subset \mathbb{R}^{N}(N \geq 3)$ is a bounded smooth domain. By using the perturbation method, we establish the existence of both positive and negative solutions for this system.

\section{Introduction}

Let us consider the following modified nonlinear Schrödinger system:

$$
\begin{gathered}
-\Delta u+u-\frac{1}{2} u \Delta\left(u^{2}\right)=\frac{2 \alpha}{\alpha+\beta}|u|^{\alpha-2}|v|^{\beta} u, \quad x \in \Omega, \\
-\Delta v+v-\frac{1}{2} v \Delta\left(v^{2}\right)=\frac{2 \beta}{\alpha+\beta}|u|^{\alpha}|v|^{\beta-2} v, \quad x \in \Omega, \\
u=0, \quad v=0, \quad x \in \partial \Omega,
\end{gathered}
$$

where $\alpha>2, \beta>2, \alpha+\beta<2 \cdot 2^{*}, 2^{*}=2 N /(N-2)$ is the critical Sobolev exponent, and $\Omega \subset \mathbb{R}^{N}(N \geq 3)$ is a bounded smooth domain.

Solutions for the system (1) are related to the existence of the standing wave solutions of the following quasilinear Schrödinger equation:

$$
\begin{array}{r}
i \partial_{t} z=-\Delta z+V(x) z-f\left(|z|^{2}\right) z-k \Delta h\left(|z|^{2}\right) h^{\prime}\left(|z|^{2}\right) z, \\
x \in \mathbb{R}^{N},
\end{array}
$$

where $V(x)$ is a given potential, $k$ is a real constant, and $f, h$ are real functions. We would like to mention that (2) appears more naturally in mathematical physics and has been derived as models of several physical phenomena corresponding to various types of $h$. For instance, the case $h(s)=s$ was used for the superfluid film equation in plasma physics by Kurihara [1] (see also [2]); in the case of $h(s)=(1+s)^{1 / 2}$, (2) was used as a model of the self-changing of a high-power ultrashort laser in matter (see [3-6] and references therein).

In recent years, much attention has been devoted to the quasilinear Schrödinger equation of the following form:

$$
-\Delta u+\lambda V(x) u-k \Delta\left(u^{2}\right) u=|u|^{p-2} u, \quad x \in \mathbb{R}^{N}
$$

See, for example, by using a constrained minimization argument, the existence of positive ground state solution was proved by Poppenberg et al. [7]. Using a change of variables, Liu et al. [8] used an Orlicz space to prove the existence of soliton solution for (3) via mountain pass theorem. Colin and Jeanjean [9] also made use of a change of variables but worked in the Sobolev space $H^{1}\left(R^{N}\right)$; they proved the existence of positive solution for (3) from the classical results given by Berestycki and Lions [10]. Liu et al. [11] established the existence of both one-sign and nodal ground states of soliton type solutions for (3) by the Nehari method. In particular, in [12], by using Nehari manifold method and concentration compactness principle (see [13]) in the Orlicz space, Guo 
and Tang considered the following quasilinear Schrödinger system:

$$
\begin{array}{r}
-\Delta u+(\lambda a(x)+1) u-\frac{1}{2}\left(\Delta|u|^{2}\right) u=\frac{2 \alpha}{\alpha+\beta}|u|^{\alpha-2}|v|^{\beta} u, \\
x \in R^{N}, \\
-\Delta u+(\lambda b(x)+1) u-\frac{1}{2}\left(\Delta|u|^{2}\right) u=\frac{2 \beta}{\alpha+\beta}|u|^{\alpha}|v|^{\beta-2} v, \\
u(x) \longrightarrow 0, \quad v(x) \longrightarrow 0, \quad|x| \longrightarrow \infty R^{N},
\end{array}
$$

with $a(x) \geq 0, b(x) \geq 0$ having a potential well and $\alpha>$ $2, \beta>2, \alpha+\beta<2 \cdot 2^{*}$, where $2^{*}=2 N /(N-2)$ is the critical Sobolev exponent, and they proved the existence of a ground state solution for the system (4) which localizes near the potential well int $a^{-1}(0)$ for $\lambda$ large enough. Guo and Tang [14] also considered ground state solutions of the single quasilinear Schrödinger equation corresponding to the system (4) by the same methods and obtained similar results.

It is worth pointing out that the existence of one-bump or multibump bound state solutions for the related semilinear Schrödinger equation (3) for $k=0$ has been extensively studied. One can see Bartsch and Wang [15], Ambrosetti et al. [16], Ambrosetti et al. [17], Byeon and Wang [18], Cingolani and Lazzo [19], Cingolani and Nolasco [20], Del Pino and Felmer [21, 22], Floer and Weinstein [23], and Oh [24, 25] and the references therein.

The system (1) is a kind of "limit" problem of the system (4) as $\lambda \rightarrow \infty$. The existence of solutions for the system (1) has important physical interest. The purpose of this paper is to study the existence of both positive and negative solutions for the system (1). We mainly follow the idea of Liu et al. [26] to perturb the functional and obtain our main results. We point out that the procedure to the system (1) is not trivial at all. Since the appearance of the quasilinear terms $u \Delta\left(u^{2}\right)$ and $v \Delta\left(v^{2}\right)$, we need more delicate estimates.

The paper is organized as follows. In Section 2, we introduce a perturbation of the functional and give our main results (Theorem 1 and 2). In Section 3, we verify the PalaisSmale condition for the perturbed functional. Section 4 is devoted to some asymptotic behavior of sequence $\left\{\left(u_{n}, v_{n}\right)\right\} \subset$ $W_{0}^{1,4}(\Omega) \times W_{0}^{1,4}(\Omega)$ and $\left\{\mu_{n}\right\} \subset(0,1]$ satisfying some conditions. Finally, our main results will be proved in Section 5 .

Throughout this paper, we will use the same $C$ to denote various generic positive constants, and we will use $o(1)$ to denote quantities that tend to 0 .

\section{Perturbation of the Functional and Main Results}

In order to obtain the desired existence of solutions for the system (1), in this section, we introduce a perturbation of the functional and give our main results.
The weak form of the system (1) is

$$
\begin{aligned}
& \int_{\Omega}\left(\left(1+u^{2}\right) \nabla u \nabla \varphi+\left(1+|\nabla u|^{2}\right) u \varphi\right) \\
& +\int_{\Omega}\left(\left(1+v^{2}\right) \nabla v \nabla \psi+\left(1+|\nabla v|^{2}\right) v \psi\right) \\
& -\frac{2 \alpha}{\alpha+\beta} \int_{\Omega}|u|^{\alpha-2}|v|^{\beta} u \varphi-\frac{2 \beta}{\alpha+\beta} \int_{\Omega}|u|^{\alpha}|v|^{\beta-2} v \psi=0, \\
& \quad(\varphi, \psi) \in C_{0}^{\infty}(\Omega) \times C_{0}^{\infty}(\Omega),
\end{aligned}
$$

which is formally the variational formulation of the following functional:

$$
\begin{aligned}
I_{0}(u, v)= & \frac{1}{2} \int_{\Omega}\left(\left(1+u^{2}\right)|\nabla u|^{2}+u^{2}\right) \\
& +\frac{1}{2} \int_{\Omega}\left(\left(1+v^{2}\right)|\nabla v|^{2}+v^{2}\right)-\frac{2}{\alpha+\beta} \int_{\Omega}|u|^{\alpha}|v|^{\beta}
\end{aligned}
$$

We may define the derivative of $I_{0}$ at $(u, v)$ in the direction of $(\varphi, \psi) \in C_{0}^{\infty}(\Omega) \times C_{0}^{\infty}(\Omega)$ as follows:

$$
\begin{aligned}
\left\langle I_{0}^{\prime}(u, v),(\varphi, \psi)\right\rangle= & \int_{\Omega}\left(1+u^{2}\right) \nabla u \nabla \varphi \\
& +\int_{\Omega}\left(1+|\nabla u|^{2}\right) u \varphi+\int_{\Omega}\left(1+v^{2}\right) \nabla v \nabla \psi \\
& +\int_{\Omega}\left(1+|\nabla v|^{2}\right) v \psi \\
& -\frac{2 \alpha}{\alpha+\beta} \int_{\Omega}|u|^{\alpha-2}|v|^{\beta} u \varphi \\
& -\frac{2 \beta}{\alpha+\beta} \int_{\Omega}|u|^{\alpha}|v|^{\beta-2} v \psi .
\end{aligned}
$$

We call that $(u, v)$ is a critical point of $I_{0}$ if $(u, v) \in$ $W_{0}^{1,2}(\Omega) \times W_{0}^{1,2}(\Omega), \int_{\Omega} u^{2}|\nabla u|^{2}<\infty, \int_{\Omega} v^{2}|\nabla v|^{2}<\infty$ and $\left\langle I_{0}^{\prime}(u, v),(\varphi, \psi)\right\rangle=0$ for all $(\varphi, \psi) \in C_{0}^{\infty}(\Omega) \times C_{0}^{\infty}(\Omega)$. That is, $(u, v)$ is a weak solution for the system (1).

When we consider the system (1) by using the classical critical point theory, we encounter the difficulties due to the lack of an appropriate working space. In general it seems there is no suitable space in which the variational functional $I_{0}$ possesses both smoothness and compactness properties. For smoothness one would need to work in a space smaller than $W_{0}^{1,2}(\Omega)$ to control the term involving the quasilinear term in the system (1), but it seems impossible to obtain bounds for $(P S)_{c}$ sequence in this setting. There have been several ideas and approaches used in recent years to overcome the difficulties such as by minimizations $[7,27]$, the Nehari 
method [11], and change of variables $[8,9]$. In this paper, we consider a perturbed functional

$$
\begin{aligned}
I_{\mu}(u, v)= & \frac{1}{4} \mu \int_{\Omega}\left(|\nabla u|^{4}+|\nabla v|^{4}\right)+I_{0}(u, v) \\
= & \frac{1}{4} \mu \int_{\Omega}\left(|\nabla u|^{4}+|\nabla v|^{4}\right)+\frac{1}{2} \int_{\Omega}\left(\left(1+u^{2}\right)|\nabla u|^{2}+u^{2}\right) \\
& +\frac{1}{2} \int_{\Omega}\left(\left(1+v^{2}\right)|\nabla v|^{2}+v^{2}\right)-\frac{2}{\alpha+\beta} \int_{\Omega}|u|^{\alpha}|v|^{\beta},
\end{aligned}
$$

where $\mu \in(0,1]$ is a parameter. Then it is easy to see that $I_{\mu}$ is a $C^{1}$-functional on $W_{0}^{1,4}(\Omega) \times W_{0}^{1,4}(\Omega)$. We also can define the derivative of $I_{\mu}$ at $(u, v)$ in the direction of $(\varphi, \psi)$ as follows:

$$
\begin{aligned}
\left\langle I_{\mu}^{\prime}(u, v),(\varphi, \psi)\right\rangle= & \mu \int_{\Omega}|\nabla u|^{2} \nabla u \nabla \varphi+\mu \int_{\Omega}|\nabla v|^{2} \nabla v \nabla \psi \\
& +\int_{\Omega}\left(1+u^{2}\right) \nabla u \nabla \varphi+\int_{\Omega}\left(1+|\nabla u|^{2}\right) u \varphi \\
& +\int_{\Omega}\left(1+v^{2}\right) \nabla v \nabla \psi+\int_{\Omega}\left(1+|\nabla v|^{2}\right) v \psi \\
& -\frac{2 \alpha}{\alpha+\beta} \int_{\Omega}|u|^{\alpha-2}|v|^{\beta} u \varphi \\
& -\frac{2 \beta}{\alpha+\beta} \int_{\Omega}|u|^{\alpha}|v|^{\beta-2} v \psi
\end{aligned}
$$

for all $(\varphi, \psi) \in C_{0}^{\infty}(\Omega) \times C_{0}^{\infty}(\Omega)$. The idea is to obtain the existence of the critical points of $I_{\mu}$ for $\mu>0$ small and to establish suitable estimates for the critical points as $\mu \rightarrow 0$ so that we may pass to the limit to get the solutions for the original system (1).

Our main results are as follows.

Theorem 1. Assume that $\alpha>2, \beta>2$ and $\alpha+\beta<2 \cdot 2^{*}$. Let $\mu_{n} \rightarrow 0$ and let $\left\{\left(u_{n}, v_{n}\right)\right\} \subset W_{0}^{1,4}(\Omega) \times W_{0}^{1,4}(\Omega)$ be a sequence of $I_{\mu_{n}}$ satisfying $I_{\mu_{n}}^{\prime}\left(u_{n}, v_{n}\right)=0$ and $I_{\mu_{n}}\left(u_{n}, v_{n}\right) \leq C$ for some $C$ independent of $n$. Then, up to a subsequence

$$
\begin{gathered}
u_{n} \longrightarrow u, \quad v_{n} \longrightarrow v \quad \text { in } W_{0}^{1,2}(\Omega), \\
u_{n} \nabla u_{n} \longrightarrow u \nabla u, \quad v_{n} \nabla v_{n} \longrightarrow v \nabla v \quad \text { in } L^{2}(\Omega), \\
\mu_{n} \int_{\Omega}\left(\left|\nabla u_{n}\right|^{4}+\left|\nabla v_{n}\right|^{4}\right) \longrightarrow 0, \\
I_{\mu_{n}}^{\prime}\left(u_{n}, v_{n}\right) \longrightarrow I_{0}^{\prime}(u, v)
\end{gathered}
$$

as $n \rightarrow \infty$ and $(u, v)$ is a critical point of $I_{0}$.

Using Theorem 1, we have the following existence result.

Theorem 2. Assume that $\alpha>2, \beta>2$ and $\alpha+\beta<2 \cdot 2^{*}$. Then $I_{\mu}$ has a positive critical point $\left(u_{\mu}, v_{\mu}\right)$ and a negative critical point $\left(\widetilde{u}_{\mu}, \widetilde{v}_{\mu}\right)$, and $\left(u_{\mu}, v_{\mu}\right)$ (resp., $\left.\left(\widetilde{u}_{\mu}, \widetilde{v}_{\mu}\right)\right)$ converges to a positive (resp., negative) solution for the system (1) as $\mu \rightarrow 0$.
Notation. We denote by $\|\cdot\|$ the norm of $W_{0}^{1,4}(\Omega)$ and by $|\cdot|_{s}$ the norm of $L^{s}(\Omega)(1 \leq s<+\infty)$.

\section{Compactness of the Perturbed Functional}

In this section, we verify the Palais-Smale condition ((PS) condition in short) for the perturbed functional $I_{\mu}(u, v)$. We have the following proposition.

Proposition 3. For $\mu>0$ fixed, the functional $I_{\mu}(u, v)$ satisfies $(P S)_{c}$ condition for all $c \in \mathbb{R}$. That is, any sequence $\left\{\left(u_{n}, v_{n}\right)\right\} \subset$ $W_{0}^{1,4}(\Omega) \times W_{0}^{1,4}(\Omega)$ satisfying, for $c \in \mathbb{R}$,

$$
\begin{gathered}
I_{\mu}\left(u_{n}, v_{n}\right) \longrightarrow c, \\
I_{\mu}^{\prime}\left(u_{n}, v_{n}\right) \longrightarrow 0 \quad \text { strongly in }\left(W_{0}^{1,4}(\Omega) \times W_{0}^{1,4}(\Omega)\right)^{*}
\end{gathered}
$$

has a strongly convergent subsequence in $W_{0}^{1,4}(\Omega) \times W_{0}^{1,4}(\Omega)$, where $\left(W_{0}^{1,4}(\Omega) \times W_{0}^{1,4}(\Omega)\right)^{*}$ is the dual space of $W_{0}^{1,4}(\Omega) \times$ $W_{0}^{1,4}(\Omega)$.

For giving the proof of Proposition 3, we need the following lemma firstly.

Lemma 4. Suppose that a sequence $\left\{\left(u_{n}, v_{n}\right)\right\} \subset W_{0}^{1,4}(\Omega) \times$ $W_{0}^{1,4}(\Omega)$ satisfies (11). Then

$$
\limsup _{n \rightarrow \infty}\left\|\left(u_{n}, v_{n}\right)\right\|^{4} \leq\left(\frac{1}{4}-\frac{1}{\alpha+\beta}\right)^{-1} \mu^{-1} c .
$$

Proof. It follows from (11) that

$$
\begin{aligned}
c+ & o(1)-\frac{1}{\alpha+\beta} o(1)\left\|\left(u_{n}, v_{n}\right)\right\| \\
= & I_{\mu}\left(u_{n}, v_{n}\right)-\frac{1}{\alpha+\beta}\left\langle I_{\mu}^{\prime}\left(u_{n}, v_{n}\right),\left(u_{n}, v_{n}\right)\right\rangle \\
= & \left(\frac{1}{4}-\frac{1}{\alpha+\beta}\right) \mu \int_{\Omega}\left(\left|\nabla u_{n}\right|^{4}+\left|\nabla v_{n}\right|^{4}\right) \\
& +\left(\frac{1}{2}-\frac{1}{\alpha+\beta}\right) \int_{\Omega}\left(\left|\nabla u_{n}\right|^{2}+\left|\nabla v_{n}\right|^{2}\right) \\
& +\left(\frac{1}{2}-\frac{1}{\alpha+\beta}\right) \int_{\Omega}\left(\left|u_{n}\right|^{2}+\left|v_{n}\right|^{2}\right) \\
& +\left(\frac{1}{2}-\frac{2}{\alpha+\beta}\right) \int_{\Omega}\left(u_{n}^{2}\left|\nabla u_{n}\right|^{2}+v_{n}^{2}\left|\nabla v_{n}\right|^{2}\right) \\
\geq & \left(\frac{1}{4}-\frac{1}{\alpha+\beta}\right) \mu \int_{\Omega}\left(|\nabla u|^{4}+|\nabla v|^{4}\right) .
\end{aligned}
$$

Thus we have

$$
\limsup _{n \rightarrow \infty}\left\|\left(u_{n}, v_{n}\right)\right\|^{4} \leq\left(\frac{1}{4}-\frac{1}{\alpha+\beta}\right)^{-1} \mu^{-1} c .
$$

This completes the proof of Lemma 4.

Now we give the proof of Proposition 3. 
Proof of Proposition 3. From Lemma 4, we know that $\left\{\left(u_{n}, v_{n}\right)\right\}$ is bounded in $W_{0}^{1,4}(\Omega) \times W_{0}^{1,4}(\Omega)$. So there exists a subsequence of $\left\{\left(u_{n}, v_{n}\right)\right\}$, still denoted $\left\{\left(u_{n}, v_{n}\right)\right\}$, such that

$\left(u_{n}, v_{n}\right) \rightarrow(u, v) \quad$ weakly in $W_{0}^{1,4}(\Omega) \times W_{0}^{1,4}(\Omega)$ as $n \longrightarrow \infty$,

$u_{n} \longrightarrow u, \quad v_{n} \longrightarrow v \quad$ strongly in $L^{s}(\Omega)$

as $n \longrightarrow \infty$ for any $2<s<2 \cdot 2^{*}$.

Now we prove that $\left(u_{n}, v_{n}\right) \rightarrow(u, v)$ in $W_{0}^{1,4}(\Omega) \times W_{0}^{1,4}(\Omega)$. In (9), choosing $(\varphi, \psi)=\left(u_{n}-u_{m}, v_{n}-v_{m}\right)$, we have

$$
\begin{aligned}
o(1) & \left\|\left(u_{n}-u_{m}, v_{n}-v_{m}\right)\right\| \\
= & \left\langle I_{\mu}^{\prime}\left(u_{n}, v_{n}\right)-I_{\mu}^{\prime}\left(u_{m}, v_{m}\right),\left(u_{n}-u_{m}, v_{n}-v_{m}\right)\right\rangle \\
= & \mu \int_{\Omega}\left(\left|\nabla u_{n}\right|^{2} \nabla u_{n}-\left|\nabla u_{m}\right|^{2} \nabla u_{m}\right)\left(\nabla u_{n}-\nabla u_{m}\right) \\
& +\mu \int_{\Omega}\left(\left|\nabla v_{n}\right|^{2} \nabla v_{n}-\left|\nabla v_{m}\right|^{2} \nabla v_{m}\right)\left(\nabla v_{n}-\nabla v_{m}\right) \\
& +\int_{\Omega}\left|u_{n}-u_{m}\right|^{2}+\int_{\Omega}\left|\nabla u_{n}-\nabla u_{m}\right|^{2} \\
& +\int_{\Omega}\left(u_{n}^{2} \nabla u_{n}-u_{m}^{2} \nabla u_{m}\right)\left(\nabla u_{n}-\nabla u_{m}\right)+\int_{\Omega}\left|v_{n}-v_{m}\right|^{2} \\
& +\int_{\Omega}\left|\nabla v_{n}-\nabla v_{m}\right|^{2}+\int_{\Omega}\left(v_{n}^{2} \nabla v_{n}-v_{m}^{2} \nabla v_{m}\right)\left(\nabla v_{n}-\nabla v_{m}\right) \\
& +\int_{\Omega}\left(u_{n}\left|\nabla u_{n}\right|^{2}-u_{m}\left|\nabla u_{m}\right|^{2}\right)\left(u_{n}-u_{m}\right) \\
& +\int_{\Omega}\left(v_{n}\left|\nabla v_{n}\right|^{2}-v_{m}\left|\nabla v_{m}\right|^{2}\right)\left(v_{n}-v_{m}\right) \\
& -\frac{2 \alpha}{\alpha+\beta} \int_{\Omega}\left(\left|u_{n}\right|^{\alpha-2}\left|v_{n}\right|^{\beta} u_{n}-\left|u_{m}\right|^{\alpha-2}\left|v_{m}\right|^{\beta} u_{m}\right)\left(u_{n}-u_{m}\right) \\
& -\frac{2 \beta}{\alpha+\beta} \int_{\Omega}\left(\left|u_{n}\right|^{\alpha}\left|v_{n}\right|^{\beta-2} v_{n}-\left|u_{m}\right|^{\alpha}\left|v_{m}\right|^{\beta-2} v_{m}\right)\left(v_{n}-v_{m}\right) .
\end{aligned}
$$

We may estimate the terms involved as follows:

$$
\begin{aligned}
& \mu \int_{\Omega}\left(\left|\nabla u_{n}\right|^{2} \nabla u_{n}-\left|\nabla u_{m}\right|^{2} \nabla u_{m}\right)\left(\nabla u_{n}-\nabla u_{m}\right) \\
& \geq \mu \int_{\Omega}\left|\nabla u_{n}-\nabla u_{m}\right|^{4}, \\
& \mu \int_{\Omega}\left(\left|\nabla v_{n}\right|^{2} \nabla v_{n}-\left|\nabla v_{m}\right|^{2} \nabla v_{m}\right)\left(\nabla v_{n}-\nabla v_{m}\right) \\
& \quad \geq \mu \int_{\Omega}\left|\nabla v_{n}-\nabla v_{m}\right|^{4},
\end{aligned}
$$

$$
\begin{aligned}
& \int_{\Omega}\left(u_{n}^{2} \nabla u_{n}-u_{m}^{2} \nabla u_{m}\right)\left(\nabla u_{n}-\nabla u_{m}\right) \\
& \quad \geq \int_{\Omega} u_{n}^{2}\left|\nabla u_{n}-\nabla u_{m}\right|^{2}+\int_{\Omega}\left(u_{n}^{2}-u_{m}^{2}\right) \nabla u_{m}\left(\nabla u_{n}-\nabla u_{m}\right) \\
& \quad \geq-\left|u_{n}-u_{m}\right|_{4}\left(\left|u_{n}\right|_{4}+\left|u_{m}\right|_{4}\right)\left\|u_{m}\right\|\left(\left\|u_{n}\right\|+\left\|u_{m}\right\|\right) \\
& \quad \longrightarrow 0 \quad \text { as } m, n \longrightarrow \infty
\end{aligned}
$$$$
\int_{\Omega}\left(v_{n}^{2} \nabla v_{n}-v_{m}^{2} \nabla v_{m}\right)\left(\nabla v_{n}-\nabla v_{m}\right)
$$$$
\geq \int_{\Omega} v_{n}^{2}\left|\nabla v_{n}-\nabla v_{m}\right|^{2}+\int_{\Omega}\left(v_{n}^{2}-v_{m}^{2}\right) \nabla v_{m}\left(\nabla v_{n}-\nabla v_{m}\right)
$$$$
\geq-\left|v_{n}-v_{m}\right|_{4}\left(\left|v_{n}\right|_{4}+\left|v_{m}\right|_{4}\right)\left\|v_{m}\right\|\left(\left\|v_{n}\right\|+\left\|v_{m}\right\|\right)
$$$$
\longrightarrow 0 \text { as } m, n \longrightarrow \infty
$$$$
\left|\int_{\Omega}\left(u_{n}\left|\nabla u_{n}\right|^{2}-u_{m}\left|\nabla u_{m}\right|^{2}\right)\left(u_{n}-u_{m}\right)\right|
$$$$
\leq\left(\left|u_{n}\right|_{4}\left\|u_{n}\right\|^{2}+\left|u_{m}\right|_{4}\left\|u_{m}\right\|^{2}\right)\left|u_{n}-u_{m}\right|_{4}
$$$$
\longrightarrow 0 \text { as } m, n \longrightarrow \infty \text {, }
$$

$$
\begin{aligned}
& \left|\int_{\Omega}\left(v_{n}\left|\nabla v_{n}\right|^{2}-v_{m}\left|\nabla v_{m}\right|^{2}\right)\left(v_{n}-v_{m}\right)\right| \\
& \quad \leq\left(\left|v_{n}\right|_{4}\left\|v_{n}\right\|^{2}+\left|v_{m}\right|_{4}\left\|v_{m}\right\|^{2}\right)\left|v_{n}-v_{m}\right|_{4} \\
& \quad \longrightarrow 0 \text { as } m, n \longrightarrow \infty
\end{aligned}
$$

$$
\begin{aligned}
& \frac{2 \alpha}{\alpha+\beta}\left|\int_{\Omega}\left(\left|u_{n}\right|^{\alpha-2}\left|v_{n}\right|^{\beta} u_{n}-\left|u_{m}\right|^{\alpha-2}\left|v_{m}\right|^{\beta} u_{m}\right)\left(u_{n}-u_{m}\right)\right| \\
& \quad \leq \frac{2 \alpha}{\alpha+\beta} \int_{\Omega}\left(\left|u_{n}\right|^{\alpha-1}\left|v_{n}\right|^{\beta}+\left|u_{m}\right|^{\alpha-1}\left|v_{m}\right|^{\beta}\right)\left|u_{n}-u_{m}\right| \\
& \quad \leq \frac{2 \alpha}{\alpha+\beta}\left(\left|u_{n}\right|_{\alpha+\beta}^{\alpha-1}\left|v_{n}\right|_{\alpha+\beta}^{\beta}+\left|u_{m}\right|_{\alpha+\beta}^{\alpha-1}\left|v_{m}\right|_{\alpha+\beta}^{\beta}\right)\left|u_{n}-u_{m}\right|_{\alpha+\beta}
\end{aligned}
$$$$
\longrightarrow 0 \text { as } m, n \longrightarrow \infty \text {, }
$$$$
\frac{2 \beta}{\alpha+\beta}\left|\int_{\Omega}\left(\left|u_{n}\right|^{\alpha}\left|v_{n}\right|^{\beta-2} v_{n}-\left|u_{m}\right|^{\alpha}\left|v_{m}\right|^{\beta-2} v_{m}\right)\left(v_{n}-v_{m}\right)\right|
$$$$
\leq \frac{2 \beta}{\alpha+\beta} \int_{\Omega}\left(\left|u_{n}\right|^{\alpha}\left|v_{n}\right|^{\beta-1}+\left|u_{m}\right|^{\alpha}\left|v_{m}\right|^{\beta-1}\right)\left|v_{n}-v_{m}\right|
$$$$
\leq \frac{2 \beta}{\alpha+\beta}\left(\left|u_{n}\right|_{\alpha+\beta}^{\alpha}\left|v_{n}\right|_{\alpha+\beta}^{\beta-1}+\left|u_{m}\right|_{\alpha+\beta}^{\alpha}\left|v_{m}\right|_{\alpha+\beta}^{\beta-1}\right)\left|v_{n}-v_{m}\right|_{\alpha+\beta}
$$$$
\longrightarrow 0 \quad \text { as } m, n \longrightarrow \infty
$$

Returning to (16), we have

$$
\begin{aligned}
& \mu \int_{\Omega}\left(\left|\nabla u_{n}-\nabla u_{m}\right|^{4}+\left|\nabla v_{n}-\nabla v_{m}\right|^{4}\right) \\
& \quad \leq o(1)\left\|\left(u_{n}-u_{m}, v_{n}-v_{m}\right)\right\|+o(1),
\end{aligned}
$$


which implies that $\left\|\left(u_{n}-u_{m}, v_{n}-v_{m}\right)\right\| \rightarrow 0$, that is, $\left(u_{n}, u_{m}\right) \rightarrow(u, v)$ in $W_{0}^{1,4}(\Omega) \times W_{0}^{1,4}(\Omega)$. This completes the proof of Proposition 3.

\section{Some Asymptotic Behavior}

Proposition 3 enables us to apply minimax argument to the functional $I_{\mu}(u, v)$. In this section, we also study the behavior of sequence $\left\{\left(u_{n}, v_{n}\right)\right\} \subset W_{0}^{1,4}(\Omega) \times W_{0}^{1,4}(\Omega)$ and $\left\{\mu_{n}\right\} \subset(0,1]$ satisfying

$$
\begin{gathered}
\mu_{n} \longrightarrow 0, \quad I_{\mu_{n}}\left(u_{n}, v_{n}\right) \longrightarrow c, \\
\left\|I_{\mu_{n}}^{\prime}\left(u_{n}, v_{n}\right)\right\|^{*} \longrightarrow 0 .
\end{gathered}
$$

The following proposition is the key of this section.

Proposition 5. Assume sequence $\left\{\left(u_{n}, v_{n}\right)\right\} \subset W_{0}^{1,4}(\Omega) \times$ $W_{0}^{1,4}(\Omega)$ and $\left\{\mu_{n}\right\} \subset(0,1]$ satisfy $(19)$. Then after extracting a sequence, still denoted by $n$, one has

$$
\begin{gathered}
\left(u_{n}, v_{n}\right)-(u, v) \quad \text { in } W_{0}^{1,2}(\Omega) \times W_{0}^{1,2}(\Omega), \\
\left(u_{n} \nabla u_{n}, v_{n} \nabla v_{n}\right) \rightarrow(u \nabla u, v \nabla v) \quad \text { in } L^{2}(\Omega) \times L^{2}(\Omega), \\
\left(u_{n}(x), v_{n}(x)\right) \longrightarrow(u(x), v(x)) \quad \text { a.e. } x \in \Omega
\end{gathered}
$$

as $n \rightarrow \infty$.

Proof. Similar to the proof of Lemma 4, by (19), we have

$$
\begin{aligned}
C \geq & I_{\mu_{n}}\left(u_{n}, v_{n}\right)-\frac{1}{\alpha+\beta}\left\langle I_{\mu_{n}}^{\prime}\left(u_{n}, v_{n}\right),\left(u_{n}, v_{n}\right)\right\rangle \\
\geq & \left(\frac{1}{4}-\frac{1}{\alpha+\beta}\right) \mu_{n} \int_{\Omega}\left(\left|\nabla u_{n}\right|^{4}+\left|\nabla v_{n}\right|^{4}\right) \\
& +\left(\frac{1}{2}-\frac{1}{\alpha+\beta}\right) \int_{\Omega}\left(\left|\nabla u_{n}\right|^{2}+\left|\nabla v_{n}\right|^{2}\right) \\
& +\left(\frac{1}{2}-\frac{1}{\alpha+\beta}\right) \int_{\Omega}\left(\left|u_{n}\right|^{2}+\left|v_{n}\right|^{2}\right) \\
& +\left(\frac{1}{2}-\frac{2}{\alpha+\beta}\right) \int_{\Omega}\left(u_{n}^{2}\left|\nabla u_{n}\right|^{2}+v_{n}^{2}\left|\nabla v_{n}\right|^{2}\right) .
\end{aligned}
$$

Thus

$$
\begin{aligned}
& \mu_{n} \int_{\Omega}\left(\left|\nabla u_{n}\right|^{4}+\left|\nabla v_{n}\right|^{4}\right)+\int_{\Omega}\left(\left|\nabla u_{n}\right|^{2}+\left|\nabla v_{n}\right|^{2}\right) \\
& \quad+\int_{\Omega}\left(\left|u_{n}\right|^{2}+\left|v_{n}\right|^{2}\right)+\int_{\Omega}\left(u_{n}^{2}\left|\nabla u_{n}\right|^{2}+v_{n}^{2}\left|\nabla v_{n}\right|^{2}\right) \leq C
\end{aligned}
$$

for some $C$ independent of $n$. Then, up to a subsequence, we have

$$
\begin{gathered}
\left(u_{n}, v_{n}\right) \rightarrow(u, v) \quad \text { in } W_{0}^{1,2}(\Omega) \times W_{0}^{1,2}(\Omega), \\
\left(u_{n} \nabla u_{n}, v_{n} \nabla v_{n}\right) \rightarrow(u \nabla u, v \nabla v) \quad \text { in } L^{2}(\Omega) \times L^{2}(\Omega), \\
\left(u_{n}(x), v_{n}(x)\right) \longrightarrow(u(x), v(x)) \quad \text { a.e. } x \in \Omega
\end{gathered}
$$

as $n \rightarrow \infty$. This completes the proof of Proposition 5.

\section{Proof of Main Results}

In this section, we give the proof of our main results. Firstly, we prove Theorem 1.

Proof of Theorem 1. Note that $\left(u_{n}, v_{n}\right)$ satisfies the following equation:

$$
\begin{aligned}
& \mu_{n} \int_{\Omega}\left|\nabla u_{n}\right|^{2} \nabla u_{n} \nabla \varphi+\mu_{n} \int_{\Omega}\left|\nabla v_{n}\right|^{2} \nabla v_{n} \nabla \psi \\
& +\int_{\Omega}\left(\left(1+u_{n}^{2}\right) \nabla u_{n} \nabla \varphi+\left(1+\left|\nabla u_{n}\right|^{2}\right) u_{n} \varphi\right) \\
& +\int_{\Omega}\left(\left(1+v_{n}^{2}\right) \nabla v_{n} \nabla \psi+\left(1+\left|\nabla v_{n}\right|^{2}\right) v_{n} \psi\right) \\
& -\frac{2 \alpha}{\alpha+\beta} \int_{\Omega}\left|u_{n}\right|^{\alpha-2}\left|v_{n}\right|^{\beta} u_{n} \varphi \\
& -\frac{2 \beta}{\alpha+\beta} \int_{\Omega}\left|u_{n}\right|^{\alpha}\left|v_{n}\right|^{\beta-2} v_{n} \psi=0
\end{aligned}
$$

for all $(\varphi, \psi) \in W_{0}^{1,4}(\Omega) \times W_{0}^{1,4}(\Omega)$. Since

$$
\begin{aligned}
& \left(\int_{\Omega}\left|u_{n}\right|^{4 N /(N-2)}\right)^{(N-2) / N} \leq C \int_{\Omega} u_{n}^{2}\left|\nabla u_{n}\right|^{2} \leq C, \\
& \left(\int_{\Omega}\left|v_{n}\right|^{4 N /(N-2)}\right)^{(N-2) / N} \leq C \int_{\Omega} v_{n}^{2}\left|\nabla v_{n}\right|^{2} \leq C .
\end{aligned}
$$

By Moser's iteration, we have

$$
\left|u_{n}\right|_{L^{\infty}} \leq C, \quad\left|v_{n}\right|_{L^{\infty}} \leq C .
$$

Hence,

$$
|u|_{L^{\infty}} \leq C, \quad|v|_{L^{\infty}} \leq C
$$

for some $C$ independent of $n$. To show that $(u, v)$ is a critical point of $I_{0}$ we use some arguments in $[28,29]$ (see more references therein). In (24) we choose $\varphi=\xi \exp \left(-u_{n}\right), \psi=$ $\eta \exp \left(-v_{n}\right)$, where $\xi \in C_{0}^{\infty}(\Omega), \xi \geq 0, \eta \in C_{0}^{\infty}(\Omega), \eta \geq 0$. Substituting $(\varphi, \psi)$ into $(24)$, we have

$$
\begin{aligned}
0= & \mu_{n} \int_{\Omega}\left|\nabla u_{n}\right|^{2} \nabla u_{n}\left(\nabla \xi \exp \left(-u_{n}\right)-\xi \nabla u_{n} \exp \left(-u_{n}\right)\right) \\
& +\mu_{n} \int_{\Omega}\left|\nabla v_{n}\right|^{2} \nabla v_{n}\left(\nabla \eta \exp \left(-v_{n}\right)-\eta \nabla v_{n} \exp \left(-v_{n}\right)\right) \\
& +\int_{\Omega}\left(1+u_{n}^{2}\right) \nabla u_{n}\left(\nabla \xi \exp \left(-u_{n}\right)-\xi \nabla u_{n} \exp \left(-u_{n}\right)\right) \\
& +\int_{\Omega}\left(1+v_{n}^{2}\right) \nabla v_{n}\left(\nabla \eta \exp \left(-v_{n}\right)-\eta \nabla v_{n} \exp \left(-v_{n}\right)\right) \\
& +\int_{\Omega}\left(1+\left|\nabla u_{n}\right|^{2}\right) u_{n} \xi \exp \left(-u_{n}\right)
\end{aligned}
$$




$$
\begin{aligned}
& +\int_{\Omega}\left(1+\left|\nabla v_{n}\right|^{2}\right) v_{n} \eta \exp \left(-v_{n}\right) \\
& -\frac{2 \alpha}{\alpha+\beta} \int_{\Omega}\left|u_{n}\right|^{\alpha-2}\left|v_{n}\right|^{\beta} u_{n} \xi \exp \left(-u_{n}\right) \\
& -\frac{2 \beta}{\alpha+\beta} \int_{\Omega}\left|u_{n}\right|^{\alpha}\left|v_{n}\right|^{\beta-2} v_{n} \eta \exp \left(-v_{n}\right) \\
& \leq \mu_{n} \int_{\Omega}\left|\nabla u_{n}\right|^{2} \nabla u_{n} \nabla \xi \exp \left(-u_{n}\right) \\
& +\mu_{n} \int_{\Omega}\left|\nabla v_{n}\right|^{2} \nabla v_{n} \nabla \eta \exp \left(-v_{n}\right) \\
& +\int_{\Omega}\left(1+u_{n}^{2}\right) \nabla u_{n} \nabla \xi \exp \left(-u_{n}\right) \\
& +\int_{\Omega}\left(1+v_{n}^{2}\right) \nabla v_{n} \nabla \eta \exp \left(-v_{n}\right) \\
& +\int_{\Omega} u_{n} \xi \exp \left(-u_{n}\right)+\int_{\Omega} v_{n} \eta \exp \left(-v_{n}\right) \\
& -\int_{\Omega}\left(1+u_{n}^{2}-u_{n}\right)\left|\nabla u_{n}\right|^{2} \xi \exp \left(-u_{n}\right) \\
& -\int_{\Omega}\left(1+v_{n}^{2}-v_{n}\right)\left|\nabla v_{n}\right|^{2} \eta \exp \left(-v_{n}\right) \\
& -\frac{2 \alpha}{\alpha+\beta} \int_{\Omega}\left|u_{n}\right|^{\alpha-2}\left|v_{n}\right|^{\beta} u_{n} \xi \exp \left(-u_{n}\right) \\
& -\frac{2 \beta}{\alpha+\beta} \int_{\Omega}\left|u_{n}\right|^{\alpha}\left|v_{n}\right|^{\beta-2} v_{n} \eta \exp \left(-v_{n}\right) \text {. }
\end{aligned}
$$

Note that $1+u_{n}^{2}-u_{n} \geq 0,1+v_{n}^{2}-v_{n} \geq 0$. By Fatou's Lemma, the weak convergence of $\left\{\left(u_{n}, v_{n}\right)\right\}$ and the fact that $\mu_{n} \int_{\Omega}\left(\left|\nabla u_{n}\right|^{4}+\right.$ $\left.\left|\nabla v_{n}\right|^{4}\right)$ is bounded, we have

$$
\begin{aligned}
0 \leq & \int_{\Omega}\left(1+u^{2}\right) \nabla u \nabla \xi \exp (-u)+\int_{\Omega}\left(1+v^{2}\right) \nabla v \nabla \eta \exp (-v) \\
& +\int_{\Omega} u \xi \exp (-u)+\int_{\Omega} v \eta \exp (-v) \\
& -\int_{\Omega}\left(1+u^{2}-u\right)|\nabla u|^{2} \xi \exp (-u) \\
& -\int_{\Omega}\left(1+v^{2}-v\right)|\nabla v|^{2} \eta \exp (-v) \\
& -\frac{2 \alpha}{\alpha+\beta} \int_{\Omega}|u|^{\alpha-2}|v|^{\beta} u \xi \exp (-u) \\
& -\frac{2 \beta}{\alpha+\beta} \int_{\Omega}|u|^{\alpha}|v|^{\beta-2} v \eta \exp (-v)
\end{aligned}
$$

$$
\begin{aligned}
= & \int_{\Omega}\left(1+u^{2}\right) \nabla u \nabla(\xi \exp (-u)) \\
& +\int_{\Omega}\left(1+v^{2}\right) \nabla v \nabla(\eta \exp (-v)) \\
& +\int_{\Omega}\left(1+|\nabla u|^{2}\right) u \xi \exp (-u)+\int_{\Omega}\left(1+|\nabla v|^{2}\right) v \eta \exp (-v) \\
& -\frac{2 \alpha}{\alpha+\beta} \int_{\Omega}|u|^{\alpha-2}|v|^{\beta} u \xi \exp (-u) \\
& -\frac{2 \beta}{\alpha+\beta} \int_{\Omega}|u|^{\alpha}|v|^{\beta-2} v \eta \exp (-v) .
\end{aligned}
$$

Let $(\chi, \omega) \geq(0,0),(\chi, \omega) \in C_{0}^{\infty}(\Omega) \times C_{0}^{\infty}(\Omega)$. We may choose a sequence of nonnegative functions $\left\{\left(\xi_{n}, \eta_{n}\right)\right\} \subset$ $C_{0}^{\infty}(\Omega) \times C_{0}^{\infty}(\Omega)$ such that $\left(\xi_{n}, \eta_{n}\right) \rightarrow(\chi \exp u, \omega \exp v)$ in $W_{0}^{1,2}(\Omega) \times W_{0}^{1,2}(\Omega),\left(\xi_{n}, \eta_{n}\right) \rightarrow(\chi \exp u, \omega \exp v)$ a.e. $x \in \Omega$ and $\left\{\left(\xi_{n}, \eta_{n}\right)\right\}$ is uniformly bounded in $L^{\infty}(\Omega) \times L^{\infty}(\Omega)$. Then by approximations in (29) we may obtain

$$
\begin{aligned}
& \int_{\Omega}\left(1+u^{2}\right) \nabla u \nabla \chi+\int_{\Omega}\left(1+v^{2}\right) \nabla v \nabla \omega+\int_{\Omega}\left(1+|\nabla u|^{2}\right) u \chi \\
& +\int_{\Omega}\left(1+|\nabla v|^{2}\right) v \omega-\frac{2 \alpha}{\alpha+\beta} \int_{\Omega}|u|^{\alpha-2}|v|^{\beta} u \chi \\
& -\frac{2 \beta}{\alpha+\beta} \int_{\Omega}|u|^{\alpha}|v|^{\beta-2} v \omega \geq 0
\end{aligned}
$$

for all $(\chi, \omega) \geq(0,0),(\chi, \omega) \in C_{0}^{\infty}(\Omega) \times C_{0}^{\infty}(\Omega)$. have

Similarly, we may obtain an opposite inequality. Thus we

$$
\begin{aligned}
& \int_{\Omega}\left(1+u^{2}\right) \nabla u \nabla \chi+\int_{\Omega}\left(1+v^{2}\right) \nabla v \nabla \omega+\int_{\Omega}\left(1+|\nabla u|^{2}\right) u \chi \\
& +\int_{\Omega}\left(1+|\nabla v|^{2}\right) v \omega-\frac{2 \alpha}{\alpha+\beta} \int_{\Omega}|u|^{\alpha-2}|v|^{\beta} u \chi \\
& -\frac{2 \beta}{\alpha+\beta} \int_{\Omega}|u|^{\alpha}|v|^{\beta-2} v \omega=0
\end{aligned}
$$

for all $(\chi, \omega) \in C_{0}^{\infty}(\Omega) \times C_{0}^{\infty}(\Omega)$. That is, $(u, v)$ is a critical point of $I_{0}$ and a solution for the system (1). By doing approximations again, we have $(u, v)$ in the place of $(\chi, \omega)$ of (31)

$$
\begin{aligned}
& \int_{\Omega}\left(\left(1+2 u^{2}\right)|\nabla u|^{2}+u^{2}\right)+\int_{\Omega}\left(\left(1+2 v^{2}\right)|\nabla v|^{2}+v^{2}\right) \\
& -2 \int_{\Omega}|u|^{\alpha}|v|^{\beta}=0 .
\end{aligned}
$$


Setting $(\varphi, \psi)=\left(u_{n}, v_{n}\right)$ in $(24)$, we have

$$
\begin{aligned}
& \mu_{n} \int_{\Omega}\left(\left|\nabla u_{n}\right|^{4}+\left|\nabla v_{n}\right|^{4}\right)+\int_{\Omega}\left(\left(1+2 u_{n}^{2}\right)\left|\nabla u_{n}\right|^{2}+u_{n}^{2}\right) \\
& \quad+\int_{\Omega}\left(\left(1+2 v_{n}^{2}\right)\left|\nabla v_{n}\right|^{2}+v_{n}^{2}\right)-2 \int_{\Omega}\left|u_{n}\right|^{\alpha}\left|v_{n}\right|^{\beta}=0 .
\end{aligned}
$$

Using $\int_{\Omega}\left|u_{n}\right|^{\alpha}\left|v_{n}\right|^{\beta} \rightarrow \int_{\Omega}|u|^{\alpha}|v|^{\beta}$ as $n \rightarrow \infty$, (32), (33), and lower semicontinuity, we obtain

$$
\begin{aligned}
& \int_{\Omega}\left|\nabla u_{n}\right|^{2} \longrightarrow \int_{\Omega}|\nabla u|^{2}, \quad \int_{\Omega}\left|\nabla v_{n}\right|^{2} \longrightarrow \int_{\Omega}|\nabla v|^{2}, \\
& \int_{\Omega} u_{n}^{2}\left|\nabla u_{n}\right|^{2} \longrightarrow \int_{\Omega} u^{2}|\nabla u|^{2}, \quad \int_{\Omega} v_{n}^{2}\left|\nabla v_{n}\right|^{2} \longrightarrow \int_{\Omega} v^{2}|\nabla v|^{2}
\end{aligned}
$$

as $n \rightarrow \infty$

In particular, we have

$$
\begin{gathered}
u_{n} \longrightarrow u, \quad v_{n} \longrightarrow v \quad \text { in } W_{0}^{1,2}(\Omega), \\
u_{n} \nabla u_{n} \longrightarrow u \nabla u, \quad v_{n} \nabla v_{n} \longrightarrow v \nabla v \quad \text { in } L^{2}(\Omega), \\
\mu_{n} \int_{\Omega}\left(\left|\nabla u_{n}\right|^{4}+\left|\nabla v_{n}\right|^{4}\right) \longrightarrow 0, \\
I_{\mu_{n}}^{\prime}\left(u_{n}, v_{n}\right) \longrightarrow I_{0}^{\prime}(u, v)
\end{gathered}
$$

as $n \rightarrow \infty$. This completes the proof of Theorem 1 .

Next, we apply the mountain pass theorem to obtain existence of critical points of $I_{\mu}$. Set

$$
\begin{gathered}
\Sigma_{\rho}=\left\{(u, v) \in W_{0}^{1,4}(\Omega) \times W_{0}^{1,4}(\Omega) \mid \int_{\Omega}\left(\left(1+u^{2}\right)|\nabla u|^{2}+u^{2}\right)\right. \\
\left.+\int_{\Omega}\left(\left(1+v^{2}\right)|\nabla v|^{2}+v^{2}\right) \leq \rho^{2}\right\}
\end{gathered}
$$

for $\rho>0$.

Let us consider the functional

$$
\begin{aligned}
I_{\mu}^{+}(u, v)= & \frac{1}{4} \mu \int_{\Omega}\left(|\nabla u|^{4}+|\nabla v|^{4}\right) \\
& +\frac{1}{2} \int_{\Omega}\left(\left(1+u^{2}\right)|\nabla u|^{2}+u^{2}\right) \\
& +\frac{1}{2} \int_{\Omega}\left(\left(1+v^{2}\right)|\nabla v|^{2}+v^{2}\right) \\
& -\frac{2}{\alpha+\beta} \int_{\Omega}\left(u^{+}\right)^{\alpha}\left(v^{+}\right)^{\beta} .
\end{aligned}
$$

Here and in the following we denote $u^{+}=\max \{u, 0\}$. The functional $I_{\mu}$ satisfies $(\mathrm{PS})_{c}$ condition. Similarly, we may verify that $I_{\mu}^{+}$satisfies $(\mathrm{PS})_{c}$ condition. By $\varepsilon$-Young inequality, for any $\varepsilon>0$, there exists $C_{\varepsilon}>0$ such that

$$
\left(u^{+}\right)^{\alpha}\left(v^{+}\right)^{\beta} \leq \varepsilon\left(u^{+}\right)^{\alpha+\beta}+C_{\varepsilon}\left(v^{+}\right)^{\alpha+\beta} .
$$

Since

$$
\begin{aligned}
& \int_{\Omega}|u|^{\alpha+\beta} \leq C\left(\int_{\Omega} u^{2}|\nabla u|^{2}\right)^{(\alpha+\beta) / 4}, \\
& \int_{\Omega}|v|^{\alpha+\beta} \leq C\left(\int_{\Omega} v^{2}|\nabla v|^{2}\right)^{(\alpha+\beta) / 4} .
\end{aligned}
$$

Then

$$
\begin{aligned}
- & \frac{2}{\alpha+\beta} \int_{\Omega}\left(u^{+}\right)^{\alpha}\left(v^{+}\right)^{\beta} \\
\geq & -\frac{2}{\alpha+\beta} \varepsilon \int_{\Omega}\left(u^{+}\right)^{\alpha+\beta}-\frac{2}{\alpha+\beta} C_{\varepsilon} \int_{\Omega}\left(u^{+}\right)^{\alpha+\beta} \\
\geq & -\frac{2 C}{\alpha+\beta} \varepsilon \int_{\Omega}\left(\int_{\Omega} u^{2}|\nabla u|^{2}\right)^{(\alpha+\beta) / 4} \\
& -\frac{2 C_{\varepsilon}}{\alpha+\beta}\left(\int_{\Omega} v^{2}|\nabla v|^{2}\right)^{(\alpha+\beta) / 4} \\
\geq & -\frac{2 C}{\alpha+\beta} \varepsilon \rho^{(\alpha+\beta) / 2}-\frac{2 C_{\varepsilon}}{\alpha+\beta} \rho^{(\alpha+\beta) / 2} \\
\geq & -\frac{1}{\alpha+\beta} \rho^{2}
\end{aligned}
$$

for $\varepsilon, \rho$ small. Thus we have

$$
\begin{aligned}
& I_{\mu}^{+}(u, v) \\
& \geq \frac{1}{2} \int_{\Omega}\left(\left(1+u^{2}\right)|\nabla u|^{2}+u^{2}\right) \\
&+\frac{1}{2} \int_{\Omega}\left(\left(1+v^{2}\right)|\nabla v|^{2}+v^{2}\right)-\frac{2}{\alpha+\beta} \int_{\Omega}\left(u^{+}\right)^{\alpha}\left(v^{+}\right)^{\beta} \\
& \geq \frac{1}{2} \rho^{2}-\frac{1}{\alpha+\beta} \rho^{2}=\left(\frac{1}{2}-\frac{1}{\alpha+\beta}\right) \rho^{2}
\end{aligned}
$$

for $(u, v) \in \partial \Sigma_{\rho}$ and for $\rho>0$ small enough. Choose $(\varphi, \psi) \geq(0,0),(\chi, \omega) \in C_{0}^{\infty}(\Omega) \times C_{0}^{\infty}(\Omega)$ and $T>0$. Define a path $(g, h):[0,1] \rightarrow W_{0}^{1,4}(\Omega) \times W_{0}^{1,4}(\Omega)$ by $(g(t), h(t))=$ $(t T \varphi, t T \psi)$. When $T$ is large enough, we have

$$
\begin{gathered}
I_{\mu}^{+}(g(1), h(1))<0, \\
\int_{\Omega}\left(\left(1+g^{2}(1)\right)|\nabla g(1)|^{2}+g^{2}(1)\right) \\
+\int_{\Omega}\left(\left(1+h^{2}(1)\right)|\nabla h(1)|^{2}+h^{2}(1)\right)>\rho^{2}, \\
\sup _{t \in[0,1]} I_{\mu}^{+}(g(t), h(t)) \leq m
\end{gathered}
$$

for some $m$ independent of $\mu \in(0,1]$.

Define

$$
c_{\mu}=\inf _{(g, h) \in \Gamma} \sup _{t \in[0,1]} I_{\mu}^{+}(g(t), h(t)),
$$


where

$$
\begin{gathered}
\Gamma=\left\{(g, h) \in C\left([0,1], W_{0}^{1,4}(\Omega) \times W_{0}^{1,4}(\Omega)\right) \mid(g(0), h(0))\right. \\
=(0,0),(g(1), h(1))=(T \varphi, T \psi)\} .
\end{gathered}
$$

From the mountain pass theorem we obtain that

$$
c_{\mu} \geq\left(\frac{1}{2}-\frac{1}{\alpha+\beta}\right) \rho^{2}
$$

is a critical value of $I_{\mu}^{+}$.

Let $\left(u_{\mu}, v_{\mu}\right)$ be a critical point corresponding to $c_{\mu}$. We have $\left(u_{\mu}, v_{\mu}\right) \geq(0,0)$. Thus $\left(u_{\mu}, v_{\mu}\right)$ is a positive critical point of $I_{\mu}$ by the strong maximum principle. In summary, we have the following.

Proposition 6. There exist positive constants $\rho$ and $m$ independent of $\mu$ such that $I_{\mu}$ has a positive critical point $\left(u_{\mu}, v_{\mu}\right)$ satisfying

$$
\left(\frac{1}{2}-\frac{1}{\alpha+\beta}\right) \rho^{2} \leq I_{\mu}\left(u_{\mu}, v_{\mu}\right) \leq m .
$$

Finally, we give the proof of Theorem 2 .

Proof of Theorem 2. For a positive solution of the system (1), the proof follows from Proposition 6 and Theorem 1. A similar argument gives a negative solution of the system (1). This completes the proof of Theorem 2 .

\section{Acknowledgments}

This paper was finished while Y. Jiao visited School of Mathematical Sciences of Beijing Normal University as a visiting fellow, and she would like to express her gratitude for their hospitality during her visit. Y. Jiao is supported by the National Science Foundation of China (11161041 and 31260098) and Fundamental Research Funds for the Central Universities (zyz2012074).

\section{References}

[1] S. Kurihara, "Large-amplitude quasi-solitons in superfluid films," Journal of the Physical Society of Japan, vol. 50, no. 10, pp. 3262-3267, 1981.

[2] E. W. Laedke, K. H. Spatschek, and L. Stenflo, "Evolution theorem for a class of perturbed envelope soliton solutions," Journal of Mathematical Physics, vol. 24, no. 12, pp. 2764-2769, 1983.

[3] H. S. Brandi, C. Manus, G. Mainfray, T. Lehner, and G. Bonnaud, "Relativistic and ponderomotive self-focusing of a laser beam in a radially inhomogeneous plasma. I: paraxial approximation," Physics of Fluids B, vol. 5, no. 10, pp. 3539-3550, 1993.

[4] X. L. Chen and R. N. Sudan, "Necessary and sufficient conditions for self-focusing of short ultraintense laser pulse in underdense plasma," Physical Review Letters, vol. 70, no. 14, pp. 2082-2085, 1993.
[5] A. de Bouard, N. Hayashi, and J. C. Saut, "Global existence of small solutions to a relativistic nonlinear Schrödinger equation," Communications in Mathematical Physics, vol. 189, no. 1, pp. 73105, 1997.

[6] B. Ritchie, "Relativistic self-focusing and channel formation in laser-plasma interactions," Physical Review E, vol. 50, no. 2, pp. R687-R689, 1994.

[7] M. Poppenberg, K. Schmitt, and Z. Q. Wang, "On the existence of soliton solutions to quasilinear Schrödinger equations," Calculus of Variations and Partial Differential Equations, vol. 14, no. 3, pp. 329-344, 2002.

[8] J. Q. Liu, Y. Q. Wang, and Z. Q. Wang, "Soliton solutions for quasilinear Schrödinger equations II," Journal of Differential Equations, vol. 187, no. 2, pp. 473-493, 2003.

[9] M. Colin and L. Jeanjean, "Solutions for a quasilinear Schrödinger equation: a dual approach," Nonlinear Analysis A, vol. 56, no. 2, pp. 213-226, 2004.

[10] H. Berestycki and P. L. Lions, "Nonlinear scalar field equations. I: existence of a ground state," Archive for Rational Mechanics and Analysis, vol. 82, no. 4, pp. 313-345, 1983.

[11] J. Q. Liu, Y. Q. Wang, and Z. Q. Wang, "Solutions for quasilinear Schrödinger equations via the Nehari method," Communications in Partial Differential Equations, vol. 29, no. 5-6, pp. 879901, 2004.

[12] Y. Guo and Z. Tang, "Ground state solutions for quasilinear Schrödinger systems," Journal of Mathematical Analysis and Applications, vol. 389, no. 1, pp. 322-339, 2012.

[13] P. L. Lions, "The concentration-compactness principle in the calculus of variations. The locally compact case I," Annales de l'Institut Henri Poincaré, vol. 1, no. 2, pp. 109-145, 1984.

[14] Y. Guo and Z. Tang, "Ground state solutions for the quasilinear Schrödinger equation," Nonlinear Analysis A, vol. 75, no. 6, pp. 3235-3248, 2012.

[15] T. Bartsch and Z. Q. Wang, "Multiple positive solutions for a nonlinear Schrödinger equation," Zeitschrift für Angewandte Mathematik und Physik, vol. 51, no. 3, pp. 366-384, 2000.

[16] A. Ambrosetti, M. Badiale, and S. Cingolani, "Semiclassical states of nonlinear Schrödinger equations," Archive for Rational Mechanics and Analysis, vol. 140, no. 3, pp. 285-300, 1997.

[17] A. Ambrosetti, A. Malchiodi, and S. Secchi, "Multiplicity results for some nonlinear Schrödinger equations with potentials," Archive for Rational Mechanics and Analysis, vol. 159, no. 3, pp. 253-271, 2001.

[18] J. Byeon and Z. Q. Wang, "Standing waves with a critical frequency for nonlinear Schrödinger equations II," Calculus of Variations and Partial Differential Equations, vol. 18, no. 2, pp. 207-219, 2003.

[19] S. Cingolani and M. Lazzo, "Multiple positive solutions to nonlinear Schrödinger equations with competing potential functions," Journal of Differential Equations, vol. 160, no. 1, pp. 118-138, 2000.

[20] S. Cingolani and M. Nolasco, "Multi-peak periodic semiclassical states for a class of nonlinear Schrödinger equations," Proceedings of the Royal Society of Edinburgh A, vol. 128, no. 6, pp. 1249-1260, 1998.

[21] M. Del Pino and P. L. Felmer, "Multi-peak bound states for nonlinear Schrödinger equations," Annales de l'Institut Henri Poincaré, vol. 15, no. 2, pp. 127-149, 1998.

[22] M. del Pino and P. L. Felmer, "Semi-classical states for nonlinear Schrödinger equations," Journal of Functional Analysis, vol. 149, no. 1, pp. 245-265, 1997. 
[23] A. Floer and A. Weinstein, "Nonspreading wave packets for the cubic Schrödinger equation with a bounded potential," Journal of Functional Analysis, vol. 69, no. 3, pp. 397-408, 1986.

[24] Y. G. Oh, "On positive multi-lump bound states of nonlinear Schrödinger equations under multiple well potential," Communications in Mathematical Physics, vol. 131, no. 2, pp. 223-253, 1990.

[25] Y. G. Oh, "Existence of semiclassical bound states of nonlinear Schrödinger equations with potentials of the class $(V)_{a}$," Communications in Partial Differential Equations, vol. 13, no. 12, pp. 1499-1519, 1988.

[26] X. Q. Liu, J. Q. Liu, and Z. Q. Wang, "Quasilinear elliptic equations via perturbation method," Proceedings of the American Mathematical Society, vol. 141, no. 1, pp. 253-263, 2013.

[27] J. Liu and Z. Q. Wang, "Soliton solutions for quasilinear Schrödinger equations I," Proceedings of the American Mathematical Society, vol. 131, no. 2, pp. 441-448, 2003.

[28] A. Canino and M. Degiovanni, "Nonsmooth critical point theory and quasilinear elliptic equations," in Topological Methods in Differential Equations and Inclusions, P. Q. Montreal, Ed., vol. 472 of Nato Advanced Study Institute Series C: Mathematical and Physical Sciences, pp. 1-50, Kluwer Academic, 1995.

[29] J. Q. Liu and Z. Q. Wang, "Bifurcations for quasilinear elliptic equations II," Communications in Contemporary Mathematics, vol. 10, no. 5, pp. 721-743, 2008. 


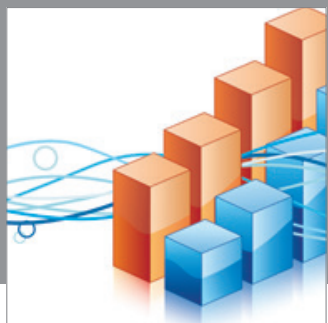

Advances in

Operations Research

mansans

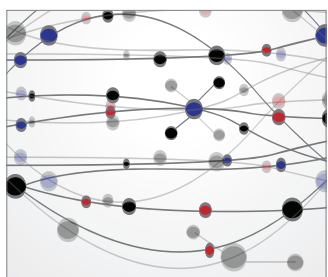

The Scientific World Journal
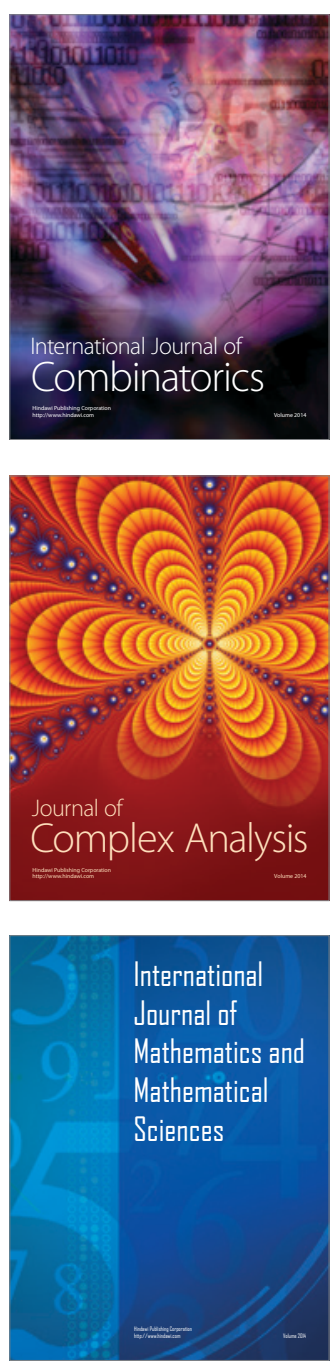
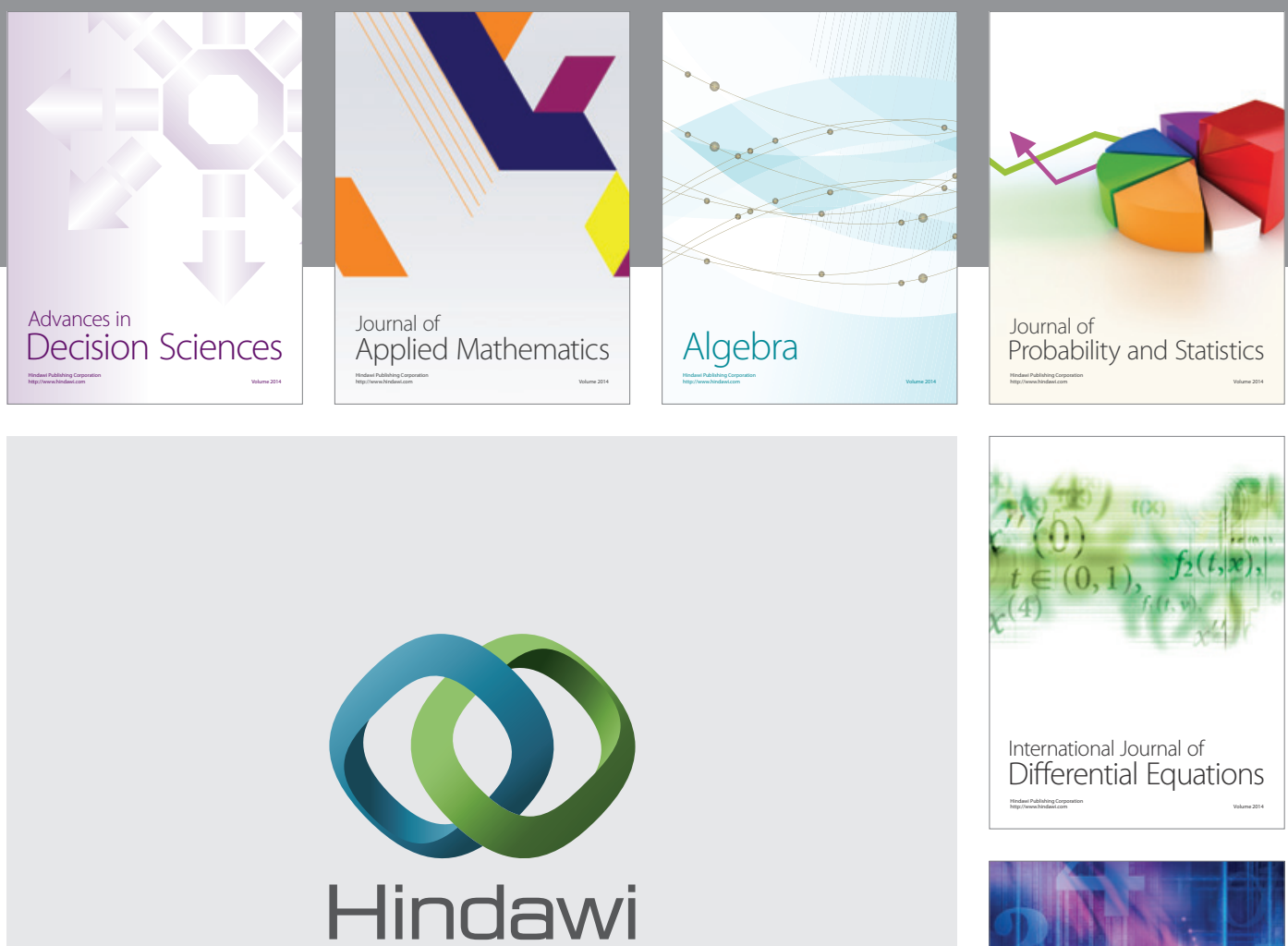

Submit your manuscripts at http://www.hindawi.com
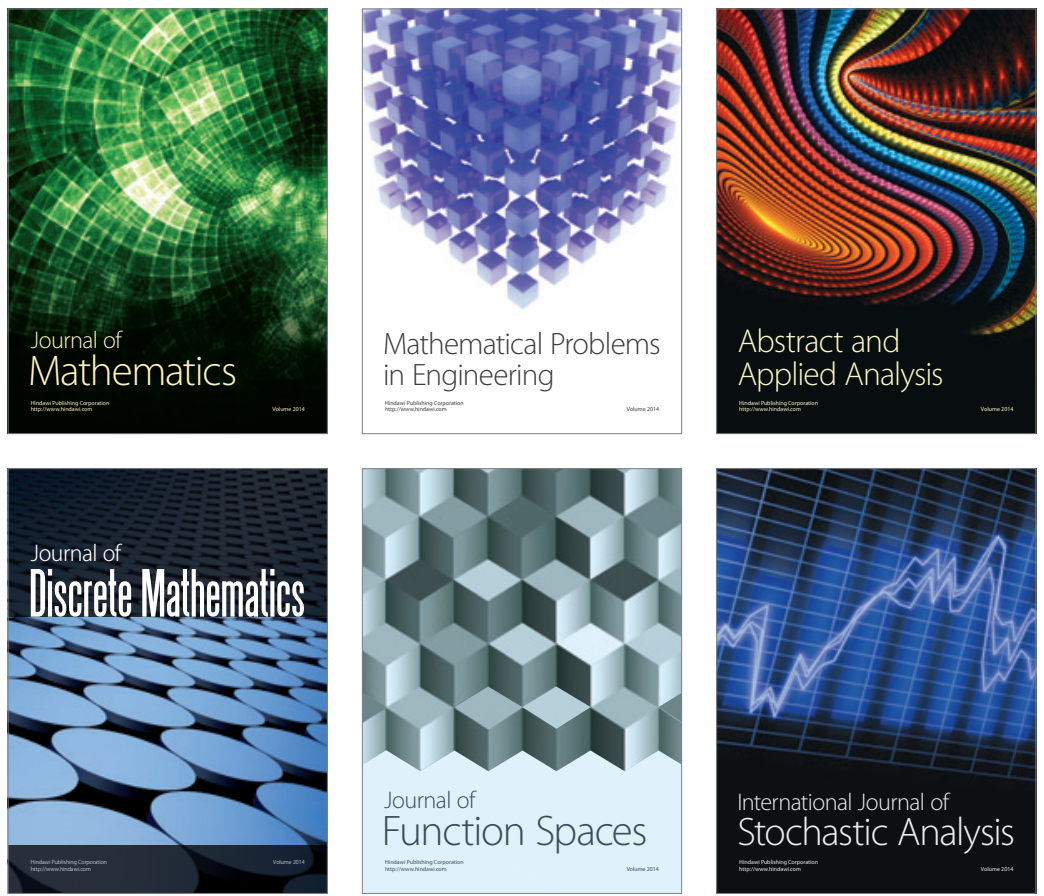

Journal of

Function Spaces

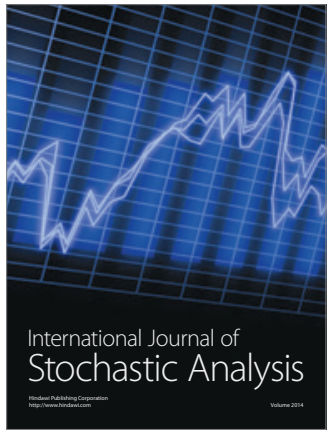

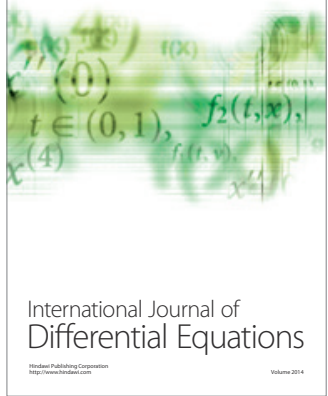
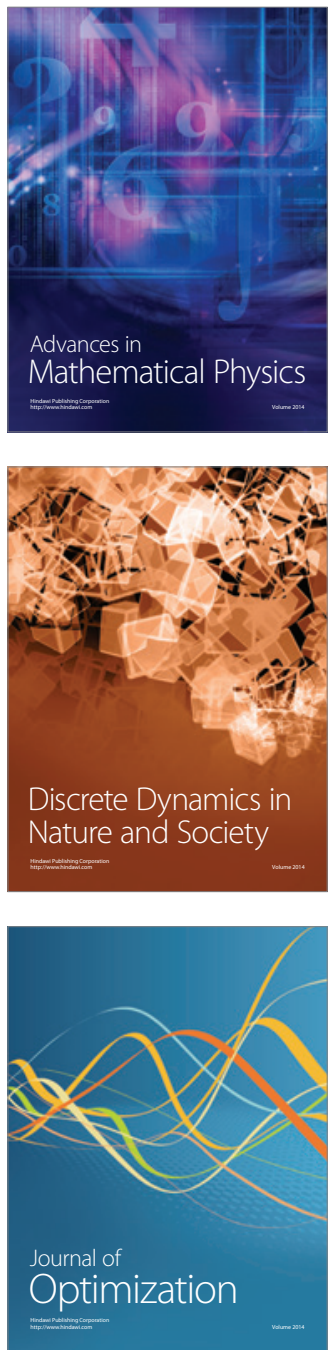